September 1995

UM-P-95/90

RCHEP-95/21

\title{
Large neutrino asymmetries from neutrino oscillations
}

\author{
R. Foot, M. J. Thomson and R. R. Volkas \\ Research Centre for High Energy Physics, \\ School of Physics, University of Melbourne, \\ Parkville, 3052 Australia.
}

\begin{abstract}
We re-examine neutrino oscillations in the early universe. Contrary to previous studies, we show that large neutrino asymmetries can arise due to oscillations between ordinary neutrinos and sterile neutrinos. This means that the Big Bang Nucleosynthesis (BBN) bounds on the mass and mixing of ordinary neutrinos with sterile neutrinos can be evaded. Also, it is possible that the neutrino asymmetries can be large (i.e. $\gtrsim 10 \%$ ), and hence have a significant effect on BBN through nuclear reaction rates.
\end{abstract}


There are several experimental indications that neutrinos have nonzero mass and oscillate [1, 2, 3]. It is possible that sterile neutrinos exist which oscillate with the known neutrinos. There are essentially two types of sterile neutrinos that can be envisaged. First, there are sterile states which either have no gauge interactions, or interactions which are much weaker than the usual weak interactions [4]. Alternatively, it is possible to envisage neutrinos which do not have significant interactions with ordinary matter but do have significant interactions with themselves. An interesting example of the latter is given by mirror neutrinos [5].

However, for both sterile and mirror neutrinos there are apparently quite stringent bounds if they are required to be consistent with standard big bang cosmology. Assuming that the effective number of neutrino species present during Big Bang Nucleosynthesis (BBN) is bounded to be less than 3.4, then the mixing angle $\left(\theta_{0}\right)$ and the squared mass difference $\left(\delta m^{2}\right)$ for a sterile neutrino mixing with one of the known neutrinos is approximately bounded by [6]

$$
\begin{gathered}
\left|\delta m^{2}\right|\left(\sin ^{2} 2 \theta_{0}\right)^{1.8} \lesssim 10^{-8} \mathrm{eV} V^{2}, \nu=\nu_{e}, \\
\left|\delta m^{2}\right|\left(\sin ^{2} 2 \theta_{0}\right)^{1.6} \lesssim 10^{-7} \mathrm{eV}^{2}, \nu=\nu_{\mu, \tau},
\end{gathered}
$$

assuming that $\delta m^{2}<0$ and $\sin ^{2} 2 \theta_{0} \lesssim 10^{-3}$ (for more precise bounds see e.g. figure 4 of Ref. [7]). These bounds arise by demanding that oscillations not bring the sterile neutrino into equilibrium with the known neutrinos. These bounds (along with even more stringent bounds for the case $\delta m^{2}>0$ or $\sin ^{2} 2 \theta_{0} \approx 1$ ) would appear to exclude the region of parameter space required to explain the atmospheric neutrino anomaly in terms of $\nu_{\mu}-\nu_{s}$ oscillation $\left(\left|\delta m^{2}\right| \simeq 10^{-2} e V^{2}\right.$, $\sin ^{2} 2 \theta_{0} \simeq 1$ ), and would restrict the parameter space required to explain the solar neutrino deficit in terms of $\nu_{e}-\nu_{s}$ oscillation. An important assumption in deriving the bounds of Eq.(1) was that the relic neutrino asymmetries could be neglected. The purpose of this paper is to re-examine this issue. We will show that for a significant region of parameter space large neutrino asymmetries can be generated due to neutrino oscillations. This has two important consequences. First, the bounds in Eq.(1) will be modified. Second, the asymmetries can be large enough to affect the neutron/proton ratio and hence also modify BBN. This is one plausible way of reconciling the possible disagreement of the BBN predictions with observations [8].

To define our notation, we first examine the ordinary neutrino $\left(\nu_{\alpha}, \alpha=e, \mu, \tau\right)$ oscillating with a sterile neutrino $\left(\nu_{s}\right)$ in vacuum. Oscillations can occur if the weak eigenstate neutrino and sterile neutrino are each linear combinations

$$
\nu_{\alpha}=\cos \theta_{0} \nu_{1}+\sin \theta_{0} \nu_{2},
$$




$$
\nu_{s}=-\sin \theta_{0} \nu_{1}+\cos \theta_{0} \nu_{2}
$$

of mass eigenstates $\nu_{1,2}$. An ordinary neutrino of momentum $p$ will then oscillate in vacuum after a time $t$ with probability

$$
\left|\left\langle\nu_{\alpha}(t) \mid \nu_{s}\right\rangle\right|^{2}=\sin ^{2} 2 \theta_{0} \sin ^{2}\left(\frac{t}{L_{o s c}}\right)
$$

where 9

$$
L_{\text {osc }}=\frac{2 p}{\delta m^{2}} \equiv \frac{1}{\Delta_{0}} .
$$

However, in the early universe oscillations occur in a plasma. For $\nu_{\alpha}-\nu_{s}$ oscillations in a plasma of temperature $T$, the matter and vacuum oscillation parameters are related by [10

$$
\begin{aligned}
& \sin ^{2} 2 \theta_{m}=\frac{\sin ^{2} 2 \theta_{0}}{1-2 z \cos 2 \theta_{0}+z^{2}}, \\
& \Delta_{m}^{2}=\Delta_{0}^{2}\left(1-2 z \cos 2 \theta_{0}+z^{2}\right),
\end{aligned}
$$

where $z=2\langle p\rangle\left\langle V_{\alpha}-V_{s}\right\rangle / \delta m^{2}$ and $\left\langle V_{\alpha, s}\right\rangle$ are the effective potentials due to the interactions of the neutrinos with matter $(\langle p\rangle \simeq 3.15 T)$. For a truly sterile neutrino $V_{s}=0$. For a weak eigenstate neutrino $\nu_{\alpha}, V_{\alpha}$ is given by [11, 12, 14]

$$
V_{\alpha}=\sqrt{2} G_{F} N_{\gamma}\left(L^{(\alpha)}-\frac{A_{\alpha} T^{2}}{M_{W}^{2}}\right),
$$

where $G_{F}$ is the Fermi coupling constant, $M_{W}$ is the $W$ boson mass, $A_{\alpha}$ is a numerical factor given by $A_{e} \simeq 55$ and $A_{\mu, \tau} \simeq 15.3$ [11, 12]. The quantity $L^{(\alpha)}$ is given by

$$
L^{(\alpha)}=L_{\alpha}+L_{\nu_{e}}+L_{\nu_{\mu}}+L_{\nu_{\tau}}+\left( \pm \frac{1}{2}+2 x_{w}\right) L_{e}+\left(\frac{1}{2}-2 x_{w}\right) L_{p}-\frac{1}{2} L_{n},
$$

where the plus sign refers to $\alpha=e$ and the minus sign refers to $\alpha=\mu, \tau$. Also, $x_{w} \equiv \sin ^{2} \theta_{w} \simeq 0.23$ and $L_{\alpha} \equiv\left(n_{\alpha}-n_{\bar{\alpha}}\right) / n_{\gamma}$.

We now study the possible creation of lepton number due to oscillations. This issue has been studied previously [12, 13] where it was concluded that significant neutrino asymmetries could not be generated due to neutrino oscillations. That conclusion was based on studies covering a limited region of parameter space (in particular for very small values of $\left|\delta m^{2}\right| \lesssim 10^{-5} \mathrm{eV}^{2}$ ). We will show below that for a large range of parameters it is possible to generate large neutrino asymmetries. Note that in Ref.13 it was observed that the neutrino asymmetry could experience a brief period of exponential growth for appropriate parameters. 
However, the particular parameters they chose led to a final asymmetry of only about $10^{-7}$. The analysis below will demonstrate that much larger asymmetries (up to $10^{-1}$ and even larger) can be generated for other interesting parameter choices.

We will work analytically, and then check our work numerically using the density matrix formalism. Our analytic treatment assumes that the change in lepton number is dominated by collisions, which is true for temperatures greater than a few $\mathrm{MeV}[15]$. We will also assume that the evolution of the neutrino ensemble follows the evolution of the state with average momentum.

The change in lepton number due to collisions can be expressed in terms of reaction rates as follows[15]:

$$
\begin{aligned}
\frac{\delta\left(n_{\nu_{\alpha}}-n_{\bar{\nu}_{\alpha}}\right)}{\delta t} & \simeq-n_{\nu_{\alpha}} \Gamma\left(\nu_{\alpha} \rightarrow \nu_{s}\right)+n_{\nu_{s}} \Gamma\left(\nu_{s} \rightarrow \nu_{\alpha}\right) \\
& +n_{\bar{\nu}_{\alpha}} \Gamma\left(\bar{\nu}_{\alpha} \rightarrow \bar{\nu}_{s}\right)-n_{\bar{\nu}_{\Gamma}} \Gamma\left(\bar{\nu}_{s} \rightarrow \bar{\nu}_{\alpha}\right)
\end{aligned}
$$

If the number densities of ordinary neutrinos are equal to the number densities of sterile neutrinos, then $\delta L_{\nu_{\alpha}} / \delta t \rightarrow 0$. This is because the rate $\Gamma\left(\nu_{\alpha} \rightarrow \nu_{s}\right)$ equals $\Gamma\left(\nu_{s} \rightarrow \nu_{\alpha}\right)$ (and similarly for the antiparticle rates). However, if the number of sterile neutrinos is negligible to start with, and if they are not brought into equilibrium with the ordinary neutrinos, then $\delta L_{\nu_{\alpha}} / \delta t$ is nonzero in general (provided $\delta m^{2}<0, \cos 2 \theta_{0}>0$, as we shall demonstrate). We thus consider parameters satisfying the bounds Eq.(1), so that the sterile neutrinos are not brought into equilibrium with the ordinary neutrinos.

Thus, assuming $n_{\nu_{s}}, n_{\bar{\nu}_{s}} \ll n_{\nu_{\alpha}}, n_{\bar{\nu}_{\alpha}}$, the change in lepton number due to collisions can be expressed as,

$$
\frac{\delta L_{\nu_{\alpha}}}{\delta t} \simeq \frac{3}{8}\left[-\Gamma\left(\nu_{\alpha} \rightarrow \nu_{s}\right)+\Gamma\left(\bar{\nu}_{\alpha} \rightarrow \bar{\nu}_{s}\right)\right]-\frac{L_{\nu_{\alpha}}}{2}\left[\Gamma\left(\nu_{\alpha} \rightarrow \nu_{s}\right)+\Gamma\left(\bar{\nu}_{\alpha} \rightarrow \bar{\nu}_{s}\right)\right],
$$

where we have used $n_{\nu_{\alpha}}=3 n_{\gamma} / 8$ [recall $L_{\nu_{\alpha}} \equiv\left(n_{\nu_{\alpha}}-n_{\bar{\nu}_{\alpha}}\right) / n_{\gamma}$ ]. It will turn out that the second term (proportional to $L_{\nu_{\alpha}}$ ) can be neglected for $T \gtrsim 1 \mathrm{MeV}$.

Thus, in order to evaluate $\delta L_{\nu_{\alpha}} / \delta t$, we need to evaluate the reaction rates. The rate $\Gamma\left(\nu_{\alpha} \rightarrow \nu_{s}\right)$ is given by the interaction rate of ordinary neutrinos multiplied by the probability that the neutrino collapses to the sterile eigenfunction, i.e.

$$
\Gamma\left(\nu_{\alpha} \rightarrow \nu_{s}\right)=\left\langle P_{\nu_{\alpha} \rightarrow \nu_{s}}\right\rangle_{\text {coll }} \Gamma_{\nu_{\alpha}}
$$

where $\Gamma_{\nu_{\alpha}}=y_{\alpha} G_{F}^{2} T^{5}$ (with $\left.y_{e} \simeq 4.0, y_{\mu, \tau} \simeq 2.9\right)$ are the reaction rates [6]. The quantity $\left\langle P_{\nu_{\alpha} \rightarrow \nu_{s}}\right\rangle_{c o l l}$ is the probability that the neutrino $\nu_{\alpha}$ collapses to the sterile state $\nu_{s}$. It is given by

$$
\left\langle P_{\nu_{\alpha} \rightarrow \nu_{s}}\right\rangle_{\text {coll }}=\sin ^{2} 2 \theta_{m}\left\langle\sin ^{2} \frac{x}{L_{o s c}^{(m)}}\right\rangle
$$


where $x$ is the distance between collisions. Note that $\langle x\rangle \equiv L_{\text {int }}=1 / \Gamma_{\nu_{\alpha}}$ where $L_{i n t}$ is the mean distance between interactions. In the region where $L_{i n t} \gg L_{o s c}^{(m)}\left(\bar{L}_{\text {osc }}^{(m)}\right),\left\langle\sin ^{2} x / L_{\text {osc }}^{(m)}\right\rangle \rightarrow 1 / 2$ and $\left\langle\sin ^{2} x / \bar{L}_{\text {osc }}^{(m)}\right\rangle \rightarrow 1 / 2\left(L_{\text {osc }}^{(m)}\right.$ and $\bar{L}_{\text {osc }}^{(m)}$ being the oscillation lengths for neutrinos and antineutrinos, respectively). Thus, evaluating $\delta L_{\nu_{\alpha}} / \delta t$ in the region where $L_{i n t} \gg L_{o s c}^{(m)}\left(\bar{L}_{o s c}^{(m)}\right)$, we find that 16

$$
\frac{\delta L_{\nu_{\alpha}}}{\delta t} \simeq \frac{3}{16} \Gamma_{\nu_{\alpha}}\left(-\sin ^{2} 2 \theta_{m_{\alpha}}+\sin ^{2} 2 \theta_{m_{\bar{\alpha}}}\right)
$$

Recall that $\sin ^{2} 2 \theta_{m_{\alpha}, m_{\bar{\alpha}}}$ are defined in Eq.(5), where $z$ is given by

$$
\begin{gathered}
z=-a+b, \text { for } \nu=\nu_{\alpha} \text { and } \\
z=a+b, \text { for } \nu=\bar{\nu}_{\alpha},
\end{gathered}
$$

where

$$
a \equiv-\sqrt{2} G_{F} n_{\gamma} L^{(\alpha)} / \Delta_{0}, b \equiv-\sqrt{2} G_{F} n_{\gamma} A_{\alpha} T^{2} /\left(\Delta_{0} M_{W}^{2}\right) .
$$

Equation (12) then becomes

$$
\frac{\delta L_{\nu_{\alpha}}}{\delta t}=\frac{3 \Gamma_{\nu_{\alpha}} \sin ^{2} 2 \theta_{0} a\left(\cos 2 \theta_{0}-b\right)}{4\left[1-2 \cos 2 \theta_{0}(-a+b)+(a-b)^{2}\right]\left[1-2 \cos 2 \theta_{0}(a+b)+(a+b)^{2}\right]} .
$$

This contribution to $\delta L_{\nu_{\alpha}} / \delta t$ is larger than the term which we neglected the second term of Eq.(9)] provided that $a /\left(1+a^{2}\right) \gg 4 L_{\nu_{\alpha}} / 3 \cos 2 \theta_{0}$, i.e. $T \gtrsim$ $0.4\left(\left|\delta m^{2}\right| / \cos 2 \theta_{0} e V^{2}\right)^{1 / 4}$. Thus, for $T \gtrsim 1 M e V$ the second term of Eq.(9) can be approximately neglected and Eq.(15) should be correct. Note that for temperatures of order $1 \mathrm{MeV}$ and below, the effect of the second term in Eq.(9) is to reduce $\left|L_{\nu_{\alpha}}\right|$.

We will assume that $\delta m^{2}<0$ (and hence $a, b>0$ ) and $\cos 2 \theta_{0}>0$ [17. Observe that for $b>\cos 2 \theta_{0}, L^{(\alpha)}=0$ is a stable fixed point. To see this, note that if $L^{(\alpha)}>0$ then $\delta L^{(\alpha)} / \delta t<0$ while for $L^{(\alpha)}<0, \delta L^{(\alpha)} / \delta t>0$. The critical observation is that when $b<\cos 2 \theta_{0}, L^{(\alpha)}=0$ becomes an unstable fixed point (i.e. if $L^{(\alpha)}>0$ then $\delta L^{(\alpha)} / \delta t>0$ while for $L^{(\alpha)}<0, \delta L^{(\alpha)} / \delta t<0$ ). Since $b$ is proportional to $T^{6}$, at some point during the evolution of the universe $b$ becomes less than $\cos 2 \theta_{0}$ and $L^{(\alpha)}=0$ becomes unstable. Observe that for $a \ll \cos 2 \theta_{0}$, $\delta L_{\nu_{\alpha}} / \delta t$ is proportional to $L_{\nu_{\alpha}}$ leading to a rapid exponential growth of $L_{\nu_{\alpha}}$.

Calculating the temperature when $b=\cos 2 \theta_{0}$, we find

$$
T_{c} \simeq 12.9(16.2)\left(\frac{\cos 2 \theta_{0}\left|\delta m^{2}\right|}{e V^{2}}\right)^{1 / 6} \mathrm{MeV}
$$


for electron (muon/tau) neutrinos.

In order to calculate the amount of $L_{\nu_{\alpha}}$ produced, it turns out that it is more convenient to use the variable $a$. This is because Eq.(15) has simple forms depending on whether $a \ll 1$ or $a \gg 1$. The quantity $\delta a / \delta t$, unlike $\delta L_{\nu_{\alpha}} / \delta t$, has a contribution from the expansion of the universe. Calculating $\delta a / \delta t$, we find,

$$
\begin{gathered}
\frac{\delta a}{\delta t}=\left.\frac{\partial a}{\partial t}\right|_{\text {exp }}+\left.\frac{\partial a}{\partial t}\right|_{o s c}=\frac{\partial T}{\partial t} \frac{\partial a}{\partial T}+\frac{\partial a}{\partial L_{\nu_{\alpha}}} \frac{\partial L_{\nu_{\alpha}}}{\partial t} \\
\simeq \frac{-5.5 T^{3}}{M_{P}} \frac{4 a}{T}-\frac{2 \sqrt{2} G_{F} n_{\gamma}}{\Delta_{0}} \frac{\delta L_{\nu_{\alpha}}}{\delta t}
\end{gathered}
$$

where we have used the time-temperature relation $t \simeq M_{P} / 11 T^{2}$ which is relevent for low temperatures (i.e. $2 m_{e}<T<m_{\mu}$ ). We have also used the result that $a$ is proportional to $T^{4}$ (so that $\partial a / \partial T=4 a / T$ ) and that $a$ is linear in $L_{\nu_{\alpha}}$. Thus, using Eq.(15) we find that

$$
\frac{\delta a}{\delta t} \simeq \frac{21 T^{2} a}{M_{P}}\left[\frac{\lambda T^{7}\left(\cos 2 \theta_{0}-b\right)}{\left[\left(\cos 2 \theta_{0}+a-b\right)^{2}+\sin ^{2} 2 \theta_{0}\right]\left[\left(\cos 2 \theta_{0}-a-b\right)^{2}+\sin ^{2} 2 \theta_{0}\right]}-1\right],
$$

where $\lambda$ is a constant given by

$$
\lambda \simeq \frac{-0.11 y_{\alpha} G_{F}^{3} \sin ^{2} 2 \theta_{0} M_{P}}{\delta m^{2}} .
$$

Note that $\lambda>0$ since we are assuming that $\delta m^{2}<0$. Observe also that $\delta a / \delta t>0$ for $0<a<a_{c}$ (where $a_{c}$ is a number greater than $\cos 2 \theta_{0}$ which we will define precisely below), provided that $T^{7} \cos 2 \theta_{0}>1 / \lambda$ for $b \ll \cos 2 \theta_{0}$ (for $b \sim \cos 2 \theta_{0}$, i.e. at resonance, the above behaviour holds for even smaller values of $T$ ). For $a \gg \cos 2 \theta_{0}, \delta a / \delta t<0$. This means that the parameter $a$ evolves towards a non-zero value: $a \rightarrow a_{c} \gtrsim \cos 2 \theta_{0}$. To calculate $a_{c}$ we solve the equation

$$
\frac{\delta a}{\delta t}=0,
$$

to find that

$$
a_{c}^{2}=\frac{K+\sqrt{K^{2}-4 C}}{2},
$$

where

$$
\begin{gathered}
K=2\left(\cos 2 \theta_{0}-b\right)^{2}-2 \sin ^{2} 2 \theta_{0}, \\
C=\left(1-2 \cos 2 \theta_{0} b+b^{2}\right)^{2}-\lambda T^{7}\left(\cos 2 \theta_{0}-b\right) .
\end{gathered}
$$


Recall that $L_{\nu_{\alpha}}$ is related to $a$ through Eq.(14). Hence, the result that $a$ evolves to $a_{c}$ is equivalent to the statement that $L_{\nu_{\alpha}}$ evolves to a non-zero value $L_{\nu_{\alpha}}^{c}$, where $L_{\nu_{\alpha}}^{c}$ is given by

$$
L_{\nu_{\alpha}}^{c}=\frac{\Delta_{0} a_{c}}{2 \sqrt{2} G_{F} n_{\gamma}} .
$$

To summarise the situation: $L_{\nu_{\alpha}}$ evolves to a very small value (so that $L^{(\alpha)} \rightarrow 0$ ) until the temperature is such that $b=\cos 2 \theta_{0}$. At this point $L^{(\alpha)}=0$ becomes an unstable fixed point and $L_{\nu_{\alpha}}$ exponentially increases until some point $a_{c} \gtrsim \cos 2 \theta_{0}$. Strictly this behaviour will not occur for all values of $\delta m^{2}, \sin ^{2} 2 \theta_{0}$. For $\sin ^{2} 2 \theta_{0}$ small enough, the evolution of $a$ will be such that it will never reach the value $a_{c}$. Nevertheless, there is a large range of parameters for which $a$ evolves to $a_{c}$.

For later times, $a_{c}$ remains greater than $\cos 2 \theta_{0}$ until

$$
\frac{\left.\delta a\right|_{\text {exp }}}{\delta t}>\max \left(\frac{\left.\delta a\right|_{\text {osc }}}{\delta t}\right) \text {. }
$$

The maximum value of $\left.\delta a\right|_{\text {osc }} / \delta t$ occurs at the resonance i.e. $a \simeq \cos 2 \theta_{0}$. Solving the above equation we find that 18

$$
T_{x} \simeq\left(\frac{9\left(1+3 \cos ^{2} 2 \theta_{0}\right)\left|\delta m^{2}\right|}{M_{P} y_{\alpha} G_{F}^{3} \cos 2 \theta_{0}}\right)^{1 / 7} .
$$

For $\cos 2 \theta_{0} \sim 1$ then

$$
T_{x} \simeq\left(\frac{\left(\left|\delta m^{2}\right| / e V^{2}\right)}{2}\right)^{1 / 7} M e V .
$$

Thus $a_{c} \geq \cos 2 \theta_{0} \sim 1$ for $T \geq T_{x}$. For later times $\left(T<T_{x}\right),\left.\delta a\right|_{\text {exp }} / \delta t>$ $\max \left(\left.\delta a\right|_{o s c} / \delta t\right)$ and $L_{\nu_{\alpha}}$ is essentially frozen. The relation $a_{c} \simeq \cos 2 \theta_{0}$ and $T=T_{x}$ implies that the corresponding value for $L_{\nu_{\alpha}}$ will be

$$
L_{\nu_{\alpha}} \simeq 2 \times 10^{-2}\left(\left|\delta m^{2}\right| / e V^{2}\right)\left(T_{x}\right)^{-4} .
$$

Recall that our analysis is really only valid for temperatures greater than about $1 \mathrm{MeV}$. For $\left|\delta m^{2}\right|<30 \mathrm{eV}^{2}$ we should take $T_{x}$ to be of order $1 \mathrm{MeV}$.

From Eq.(27) it is clear that we expect that large neutrino asymmetries can be generated. In fact, for $\left|\delta m^{2}\right| \gtrsim 5 \mathrm{eV}^{2}, L_{\nu_{\alpha}} \gtrsim 0.1$. We have checked our results numerically using the density matrix formalism (see e.g. [19] for a description of this formalism and for original references). Numerically integrating the equations describing the evolution of the density matrix we have found good agreement with the analytic results presented in this paper. In figure 1 we plot the evolution of $L_{\nu_{\alpha}}$ 
taking, by way of example, $\delta m^{2}=-1 e V^{2}, \sin ^{2} 2 \theta_{0} \simeq 10^{-8}$. Also plotted in figure 1 is the asymptotic value $L_{\nu_{e}}^{c}$ [Eq.(23)]. Figure 1 shows the behaviour expected from our analytic analysis. The lepton number $L_{\nu_{e}}$ initially grows exponentially at the resonance $b=\cos 2 \theta_{0}$ [which corresponds to $12.9 \mathrm{MeV}$ for $\delta m^{2}=-1 \mathrm{eV} V^{2}$, $\sin ^{2} 2 \theta_{0}=10^{-8}$ according to Eq.(16)]. After the exponential phase gets cut off, the neutrino asymmetry continues to grow more slowly as it approaches the asymptotic curve determined from $a_{c}$.

One important result is that the lepton number generated can be large enough to significantly modify the standard BBN scenario. Indeed if $L_{\nu_{e}}$ is positive 20] then it can reduce the neutron to proton ratio. (Such a reduction may be one way to get agreement with the data [8]).

Another important result of this work is that the bounds on ordinary neutrinos mixing with sterile neutrinos may be aleviated. We will study this issue in more detail and expand our analysis in a forthcoming article using the density matrix formalism. Below we sketch how this can happen. By way of example, assume that there is a sterile neutrino which mixes with the muon neutrino, with parameters suggested by the atmospheric neutrino anomaly (i.e. $\left|\delta m^{2}\right| \simeq 10^{-2} \mathrm{eV}^{2}$, $\left.\sin ^{2} 2 \theta_{0} \simeq 1[2]\right)$. Naively, this possibility is in conflict with BBN, because these parameters severely violate the BBN bounds of Eq.(1). However a small mixing between the tau neutrinos and the sterile neutrino may generate significant lepton number to strongly suppress the oscillations of the muon and sterile neutrino [21. This would then make this scenario consistent with BBN.

Consider a system comprised of $\nu_{\mu}, \nu_{\tau}$ and $\nu_{s}$. Note that for oscillations of tau to sterile neutrinos, the mass difference can be much bigger than the $10^{-2} \mathrm{eV}^{2}$ for muon to sterile neutrinos. $\left[\delta m_{\tau s}^{2}=m_{\nu_{s}}^{2}-m_{\nu_{\tau}}^{2} \simeq-m_{\nu_{\tau}}^{2}\right]$. Denote $b_{\tau}\left(b_{\mu}\right)$ as the value of the $b$ parameter [defined in Eq.(14)] with $\delta m^{2}=\delta m_{\tau s}^{2}\left(\delta m_{\mu s}^{2}\right)$. Because of the larger mass difference and the larger value of $\cos 2 \theta_{\tau s}$, the point $b_{\tau}=\cos 2 \theta_{\tau s}$ will be reached earlier for $\nu_{\tau}-\nu_{s}$ oscillations than the corresponding point $b_{\mu}=\cos 2 \theta_{\mu s}$ for $\nu_{\mu}-\nu_{s}$ oscillations. If the vacuum parameters for $\nu_{\tau}-\nu_{s}$ mixing, $\theta_{\tau s}, \delta m_{\tau s}^{2}$ satisfy the BBN bounds of Eq.(1), then $\nu_{s}$ cannot be brought into equilibrium by $\nu_{\tau}-\nu_{s}$ oscillation. Consequently our earlier analysis applies and we would expect significant generation of tau lepton number at this point. Note that the creation of $L_{\nu_{\tau}}$ could conceivably be compensated by $\nu_{\mu} \rightarrow \nu_{s}$ oscillations. We assume that there is significant intergenerational mixing between $\nu_{\mu}$ and $\nu_{\tau}$ which we assume rapidly converts $L_{\nu_{\tau}}$ into $L_{\nu_{\mu}}$ so that $L_{\nu_{\mu}} \simeq L_{\nu_{\tau}}$. This assumption may not really be necessary (we will study this in more detail in our forthcoming article 22]). The condition that the creation of lepton number due 
to $\nu_{\tau}-\nu_{s}$ oscillations will not be erased by $\nu_{\mu}-\nu_{s}$ oscillations is

$$
\left|\frac{\delta L_{\nu_{\tau}}}{\delta t}\right|>\left|\frac{\delta L_{\nu_{\mu}}}{\delta t}\right|
$$

for temperatures satisfing $b_{\tau}<\cos 2 \theta_{\tau s}$. Studying the above inequality away from resonance by using Eq.(15) (with $b_{\mu} / b_{\tau}=\delta m_{\tau s}^{2} / \delta m_{\mu s}^{2}$ ), we find that it is sufficient to demand that

$$
\sin ^{2} 2 \theta_{\tau s}>\sin ^{2} 2 \theta_{\mu s}\left(\frac{\delta m_{\mu s}^{2}}{\delta m_{\tau s}^{2}}\right)^{2}
$$

If $\sin ^{2} 2 \theta_{\mu s} \approx 1$ and $\delta m_{\mu s}^{2} \approx 10^{-2} e V^{2}$ then

$$
\sin ^{2} 2 \theta_{\tau s}>10^{-4} \frac{e V^{4}}{m_{\nu_{\tau}}^{4}}
$$

Thus, we arrive at a lower bound for $\sin 2 \theta_{\tau s}$, which for $m_{\nu_{\tau}}<30 \mathrm{eV}$ (as suggested by cosmology) is given by $\sin ^{2} 2 \theta_{\tau s} \gtrsim 10^{-10}$.

We also need to check that at the point where the lepton number begins to be generated [i.e. when $b_{\tau}=\cos 2 \theta_{\tau s}$, see Eq.(16)], the parameters are such that the $\nu_{s}$ have not already been brought into equilibrium. Demanding that $\frac{1}{2} \Gamma_{\nu_{\alpha}} \sin ^{2} 2 \theta_{m}<H \simeq 5.5 T^{2} / M_{P}$ for $T>T_{c}$ [defined in Eq.(16)], we find:

$$
\frac{\left|\delta m_{\mu s}^{2}\right|}{\left|\delta m_{\tau s}^{2}\right|} \lesssim \frac{\cos 2 \theta_{\tau s}}{41\left[\cos 2 \theta_{\tau s}\left|\delta m_{\tau s}^{2}\right|\right]^{\frac{1}{4}} \sin 2 \theta_{\mu s}}
$$

So, for $\left|\delta m_{\mu s}^{2}\right| \simeq 10^{-2} e V^{2}, \cos 2 \theta_{\tau s} \simeq 1$ and $\sin 2 \theta_{\mu s} \simeq 1$, we get that $m_{\nu_{\tau}} \gtrsim$ $0.5 \mathrm{eV}$.

Finally we need to check that the lepton number generated is sufficient to suppress the $\nu_{\mu}-\nu_{s}$ oscillations. Demanding that interactions not bring the sterile neutrino into equilibrium with the muon neutrino, i.e. $\Gamma_{\nu_{s}} \lesssim H \simeq 5.5 T^{2} / M_{P}$, we find in the case of large $L^{(\mu)} 21$ that

$$
\left(\delta m_{\mu s}^{2}\right)^{2} \lesssim \frac{79 G_{F}^{2} T^{2} n_{\gamma}^{2}\left[L^{(\mu)}\right]^{2}}{\frac{y_{\alpha} M_{P} G_{F}^{2} T^{3}}{11}-1},
$$

where $T>T_{d e c} \simeq 4.4 \mathrm{MeV}$ (since we only need to require that the sterile neutrinos not come into equilibrium before kinetic decoupling of the muon neutrinos occcur). Since from our earlier analysis, we expect $a_{c} \gtrsim \cos 2 \theta_{\tau s}$, we know that the $L^{(\tau)}$ generated by the $\nu_{\tau}-\nu_{s}$ oscillations satisfies

$$
\left|L^{(\tau)}\right| \gtrsim \frac{\left|\delta m_{\tau s}^{2}\right|}{2 \sqrt{2} G_{F} n_{\gamma} 6.3 T} .
$$


Thus assuming that intergenerational mixing rapidly distributes the lepton number so that $L^{(e)} \simeq L^{(\mu)} \simeq L^{(\tau)}$ then Eq.(32) is satisfied provided that

$$
\left|\delta m_{\tau s}^{2}\right| \gtrsim 10\left|\delta m_{\mu s}^{2}\right|,
$$

where the most stringent bound occurs when at the decoupling temperature i.e. $T=T_{\text {dec }} \simeq 4.4 \mathrm{MeV}$. In other words, for the parameters satisfying the above equation there is sufficient lepton number generated to prevent the sterile neutrino from coming into equilibrium (above $T_{d e c}$ ) with the muon neutrino [despite having oscillation parameters in gross violation of the bounds Eq.(1)]. Assuming $\delta m_{\tau s}^{2} \simeq-m_{\nu_{\tau}}^{2}$ and $\left|\delta m_{\mu s}^{2}\right| \simeq 10^{-2} \mathrm{eV}^{2}$ (as suggested by the atmospheric neutrino anomaly) then Eq.(34) suggests that $m_{\nu_{\tau}} \gtrsim 0.3 \mathrm{eV}$. This requirement is slightly less stringent then the $0.5 \mathrm{eV}$ bound obtained earlier from Eq.(31). Thus we conclude that muon neutrino oscillating into a sterile neutrino with maximal mixing and $\delta m^{2} \simeq 10^{-2} \mathrm{eV}^{2}$ as suggested by the atmospheric neutrino anomaly seems to be consistent with $\mathrm{BBN}$ provided that the tau neutrino oscillates into the sterile neutrinos with parameters bounded by $10^{-4} / m_{\nu_{\tau}}^{4}<\sin ^{2} 2 \theta_{\tau s} \lesssim 3 \times 10^{-5} / m_{\nu_{\tau}}^{1.2}$ [where we have used Eqs.(1) and (30)]. Note that this condition implies that $m_{\nu_{\tau}}>1.5 \mathrm{eV}$.

This result should also apply for mirror neutrinos which do not have significant interactions with ordinary particles but do have significant interactions with themselves [5]. Mirror neutrinos are required to exist if there is an unbroken parity symmetry. However, naively this scenario appears to be in conflict with BBN because it predicts that the muon neutrino will be maximally mixed with a mirror neutrino. Thus it will violate the bounds Eq.(1) if it is to be relevant for the atmospheric neutrino anomaly. However, we have shown in this paper that this scenario is not in conflict with BBN provided that the sterile neutrino also mixes slightly with the tau neutrino.

Thus we conclude that it is possible that large neutrino asymmetries can be generated by oscillations between ordinary and sterile neutrinos. This asymmetry can be very large (i.e. $\gtrsim 0.10$ ) and may thus modify nucleosynthesis. This should provide one way to reconcile the present discrepancy between the BBN predictions and observations [8]. Another consequence of a large neutrino asymmetry is that it may be possible to aleviate the BBN bounds on the mixing of ordinary and sterile neutrinos.

\section{Acknowledgements}

This work was supported by the Australian Research Council. 


\section{Figure Caption}

Electron lepton number $\left(L_{\nu_{e}}\right)$ versus temperature (in $\mathrm{MeV}$ units) for $\nu_{e}-\nu_{s}$ oscillations with parameters $\delta m^{2}=-1 \mathrm{eV}^{2}$ and $\sin ^{2} 2 \theta_{0}=10^{-8}$. The broken line is the theoretical prediction based on the numerical integration of the density matrix equations. The solid line is the asymptotic value $L_{\nu_{e}}^{c}$ [Eq.(23)].

\section{References}

[1] GALLEX Collab., Phys. Lett. B327, 377 (1994); SAGE Collab., Phys Lett. 328 B, 234 (1994). Kamiokande Collab., Nucl. Phys. B38 (Proc. Suppl.), 55 (1995); Homestake Collab., ibid, 47.

[2] K. Hirata et al, Phys. Lett. B280, 146 (1992); D. Casper et al, Phys. Rev. Lett. 66, 2561 (1991); Y. Fukuda, Phys. Lett. 335B, 237 (1994).

[3] LSND Collaboration, Preprint, Nucl-ex /9504002 (1995).

[4] See e.g. A. Yu. Smirnov and J. W. F. Valle, Nucl. Phys. B375, 649 (1992); D. O. Caldwell and R. N. Mohapatra, Phys. Rev. D48, 3259 (1993); J. Peltoniemi et al., Phys. Lett. B298, 383 (1993); A. S. Joshipura and J. W. F. Valle, hep-ph/9410259 (1994); E. Ma and P. Roy, hep-ph/9504342 (1995); Z. Berezhiani and R. N. Mohapatra, hep-ph/9505385 (1995).

[5] R. Foot, H. Lew and R. R. Volkas, Phys. Lett. B272, 67 (1991); Mod. Phys. Lett. A7, 2567 (1992); R. Foot, Mod. Phys. Lett. A9, 169 (1994); R. Foot and R. R. Volkas, University of Melbourne Preprint UM-P-95/49, hep-ph/9505359, (Phys. Rev. D, in press).

[6] A. Dolgov, Sov. J. Nucl. Phys. 33, 700 (1981); R. Barbieri and A. Dolgov, Phys. Lett. B237, 440 (1990); Nucl. Phys. B349, 743 (1991); K. Kainulainen, Phys. Lett. B244, 191 (1990); K. Enqvist, K. Kainulainen and M. Thomson, Nucl. Phys. B373, 498 (1992); J. Cline, Phys. Rev. Lett. 68, 3137 (1992); X. Shi, D. N. Schramm and B. D. Fields, Phys. Rev. D48, 2568 (1993).

[7] K. Enqvist, K. Kainulainen and M. Thomson, in Ref[6].

[8] N. Hata et. al. Ohio preprint, hep-ph/9505319 (1995).

[9] Actually $\left|L_{o s c}\right|$ is the oscillation length divided by $2 \pi$. 
[10] The propagation of neutrinos in matter was first studied by: L. Wolfenstein, Phys. Rev. D17, 2369 (1978); D20, 2634 (1979); S. P. Mikheyev and A. Yu. Smirnov, Nuovo Cim. C9, 17 (1986).

[11] D. Notzold and G. Raffelt, Nucl. Phys. B307, 924 (1988).

[12] K. Enqvist, K. Kainulainen and J. Maalampi, Nucl. Phys. B349, 754 (1991).

[13] See the second paper by Barbieri and Dolgov in Ref. [6].

[14] For antineutrinos, the equation is the same as below, except that $L^{(\alpha)} \rightarrow$ $-L^{(\alpha)}$.

[15] We can also calculate the change in lepton number due to oscillations. For $L_{\text {int }} \gg L_{\text {osc }}$ (where $\left\langle\sin ^{2} x / L_{o s c}\right\rangle \rightarrow 1 / 2$ ), the number density of neutrinos and antineutrinos is given by $n_{\nu_{\alpha}}=1-\frac{\sin ^{2} 2 \theta_{m_{\alpha}}}{2}, n_{\bar{\nu}_{\alpha}}=1-\frac{\sin ^{2} 2 \theta_{m_{\bar{\alpha}}}}{2}$ (where we are not including the depletion due to collisions). Hence,

$$
\frac{\partial\left(n_{\nu_{\alpha}}-n_{\bar{\nu}_{\alpha}}\right)}{\partial t}=\frac{\partial}{\partial t}\left(\frac{\sin ^{2} 2 \theta_{m_{\bar{\alpha}}}}{2}-\frac{\sin ^{2} 2 \theta_{m_{\alpha}}}{2}\right) .
$$

This equation has a similiar form to the contribution to $\delta L_{\nu_{\alpha}} / \delta t$ due to collisions [Eq.(15)], but it is numerically smaller provided that $y_{\alpha} G_{F}^{2} T^{5}>$ $8 T^{2} / M_{P}$ i.e. for $T \gtrsim 2 M e V$.

[16] Note that it is possible that $L_{\text {int }} / L_{\text {osc }}<1$ at the center of the resonance. Indeed,

$$
\begin{aligned}
\left(\frac{L_{\text {int }}}{L_{\text {osc }}}\right)^{2} & =\frac{\Delta_{0}^{2}\left[\left(\cos 2 \theta_{0}-(b \pm a)\right)^{2}+\sin ^{2} 2 \theta_{0}\right]}{\left(y_{\alpha} G_{F}^{2} T^{5}\right)^{2}} \\
& \approx \frac{\left(b \pm a-\cos 2 \theta_{0}\right)^{2}+\sin ^{2} 2 \theta_{0}}{\left(b^{2} / A_{\alpha}^{2}\right)}
\end{aligned}
$$

where we have used $y_{\alpha} G_{F}^{2} T^{5} / \Delta_{0} \approx b / A_{\alpha}$. However, if $\left|b \pm a-\cos 2 \theta_{0}\right| / b \gg$ $1 / A_{\alpha}$, then $L_{\text {int }} / L_{\text {osc }} \gg 1$, and hence our replacement of $\left\langle\sin ^{2} x / L_{\text {osc }}\right\rangle$ by $1 / 2$ should be valid in the resonance region, but it may not be strictly valid at the center of the resonance.

[17] Note that if we had taken $\delta m^{2}>0$, then $a, b<0$ and $L^{(\alpha)}=0$ would be a stable fixed point for all values of $a, b$ provided that $\cos 2 \theta_{0}>0$. For $\cos 2 \theta_{0}<0$ we require $\delta m^{2}>0$ for $L^{(\alpha)}=0$ to be an unstable fixed point. 
[18] Note that strictly, at resonance we may be in a region where $L_{\text {int }} / L_{\text {osc }}<$ 1 and consequently Eq.(15) is not appropriate. However recalculating $\max \left(\left.\delta a\right|_{o s c} / \delta t\right)$ in the limit where $L_{\text {int }} \ll L_{\text {osc }}$ (at the resonance) we find that $\left.\delta a\right|_{\text {exp }} / \delta t$ is always less than $\left.\delta a\right|_{\text {osc }} / \delta t$. Eventually, $L_{\text {int }}>L_{\text {osc }}$ at resonance. Thus, $T_{x}$ is given by the above, or when $L_{\text {int }} / L_{o s c} \approx 1$, which ever is later.

[19] B. H. J. McKellar and M. J. Thomson, Phys. Rev. D49, 2710 (1994).

[20] Note that we cannot predict the sign of $L^{(\alpha)}$. For temperatures where $b>$ $\cos 2 \theta_{0}, L^{(\alpha)}$ evolves very near to zero. So near to zero that for temperatures where $b<\cos 2 \theta_{0}$, it is likely that the sign of $L^{(\alpha)}$ will depend on random statistical fluctuations. It is amusing to speculate that if $L_{\nu_{e}}$ evolves to a large value so that the ratio of protons to neutrons is affected, then it may be possible that different regions of the early universe have different signs of $L_{\nu_{e}}$ and thus the ratio of protons and neutrons may not be homogeneous today!

[21] The possibility that large lepton number asymmetry can suppress transitions from ordinary to sterile neutrinos and thus weaken the BBN bounds has been studied recently by two of us, R. Foot and R. R. Volkas, University of Melbourne Preprint UM-P-95/75 (1995), hep/ph 9508275 [see also K. Enqvist, K. Kainulainen and J. Maalampi, Phys. Lett. B244, 186 (1990)]. In that paper it was assumed that the asymmetry was generated at some early time due to some unspecified mechanism. However in that paper we missed the important result that the neutrino oscillations can generate large neutrino asymmetries at relatively low temperatures (the subject of the present paper).

[22] R. Foot, M. J. Thomson and R. R. Volkas, work in progress. 


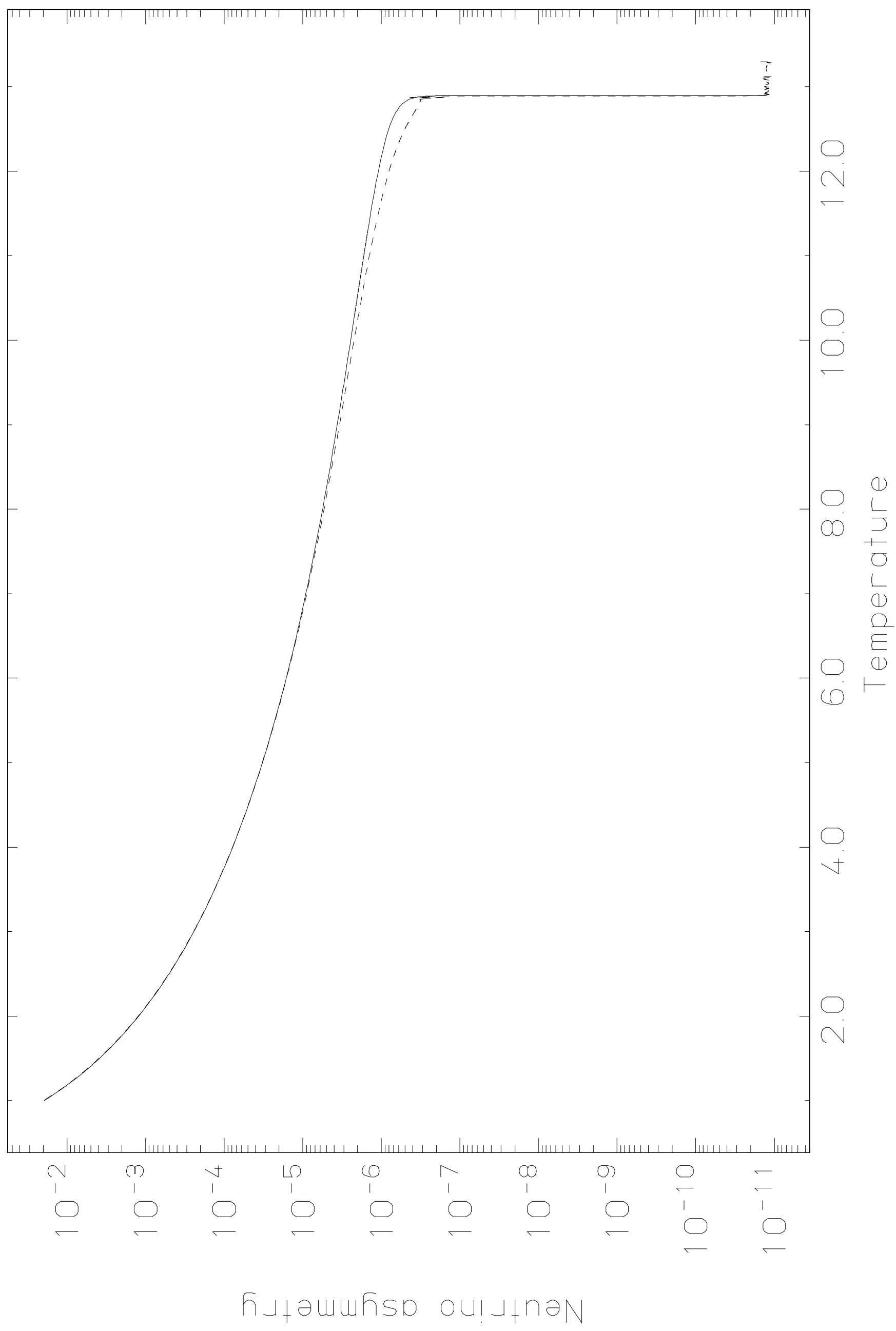

